METZLER PHILOSOPHEN LEXIKON 


\title{
METZLER \\ PHILOSOPHEN LEXIKON
}

\author{
Von den Vorsokratikern \\ bis zu den Neuen Philosophen
}

Dritte, aktualisierte und erweiterte Auflage

Herausgegeben von Bernd Lutz

Verlag J. B. Metzler

Stuttgart - Weimar 


\title{
Inhaltsverzeichnis
}

\author{
Vorwort V \\ Philosophen A-Z 1-778 \\ Weiterführende Bibliographie 779 \\ Verzeichnis der Autorinnen und Autoren 782 \\ Personenregister 784
}

Bibliografische Information Der Deutschen Bibliothek

Die Deutsche Bibliothek verzeichnet diese Publikation in der Deutschen Nationalbibliografie; detaillierte bibliografische Daten sind im Internet über `http://dnb.ddb.de` abrufbar.

\author{
ISBN 978-3-476-01953-0 \\ ISBN 978-3-476-05009-0 (eBook) \\ DOI 10.1007/978-3-476-05009-0
}

Dieses Werk einschließlich aller seiner Teile ist urheberrechtlich geschützt. Jede Verwertung außerhalb der engen Grenzen des Urheberrechtsgesetzes ist ohne Zustimmung des Verlages unzulässig und strafbar. Das gilt insbesondere für Vervielfältigungen, Übersetzungen, Mikroverfilmungen und die Einspeicherung und Verarbeitung in elektronischen Systemen.

(C) 2003 Springer-Verlag GmbH Deutschlad

Ursprünglich erschienen bei J. B. Metzlersche Verlagsbuchhandlung

und Carl Ernst Poeschel Verlag GmbH in Stuttgart 2003

www.metzlerverlag.de

info@metzlerverlag.de 


\section{Vorwort zur dritten Auflage}

Als dieses Buch erstmals 1988 erschien, war es im Kern als übergreifendes Informationsmittel für den studentischen Gebrauch konzipiert. Dennoch wurden die Artikel nicht nach dem lexikalisch üblichen Schema >Leben-Werk-Wirkung-Würdigungr aufgebaut, obwohl es gelegentlich durchscheinen mag. Schematismus sollte vermieden werden. Ein erzählerischer Einfall, eine zeitgemäße Aktualisierung sollte die Artikel tragen, Vergegenwärtigung auch des zeitlich scheinbar Entlegenen sollte erreicht werden. Immerhin reicht die Zeitspanne, die das Lexikon mit seinen 360 Artikeln abdeckt - um zwei bildliche Vorstellungen heranzuziehen - von der antiken Erdkarte des Hekataios (um 500 v. Chr.), die vom fremden Element Wasser, dem letztlich aber doch vertrauten Okeanos als eines einenden Kosmos umfaßt wird, bis zu den heutigen Momentaufnahmen einer globalisierten, sinnlos zerstörten natürlichen Umwelt des Menschen und der befremdlich bedrückenden Aufnahme der Erde als einer von Atmosphäre umgebenen Kugel, in den unendlichen Weiten eines mathematisch-physikalisch kaum vertrauten Universums schwebend. Dazwischen liegen zweieinhalb Jahrtausende der Entwicklung menschlicher Zivilisation und damit auch der verstehenden, der kritischen oder skeptischen Verständigung über sie, diskursiver Gegenstand dieses Buchs.

Mittlerweile hat sich das Buch einen weit über die studentischen Adressaten hinaus reichenden, am Gang der Philosophie interessierten Leser- und Freundeskreis erobert und wird als ein Standardwerk geachtet. Dank dieser Tatsache kann nun die dritte, aktualisierte und erweiterte Auflage vorgelegt werden. Sie hat sich äußerlich ein wenig verändert. Um angesichts der Vielzahl der neuen Artikel Platz zu sparen und die Lesbarkeit zu erhöhen, wurde die Seite zweispaltig angeordnet. Auf Porträts mußte nicht nur aus Kostengründen ganz verzichtet werden. Es gab noch einen anderen Grund: Unser medial geschärfter Blick für das Authentische ließ eine Vielzahl der Abbildungen in der ersten und in der zweiten Auflage gerade da, wo nicht mit den Mitteln der zeitgenössischen Porträtkunst oder der Photographie gearbeitet worden war, rasch verblassen.

Gegenüber der zweiten Auflage ist das Buch um rund 60 Artikel vermehrt worden. Dabei kam es nicht nur darauf an, die Gegenwartsphilosophie stärker zu berücksichtigen (rund 30 neue Artikel). Als ebenso wichtig erschien es, das historische Beziehungsgeflecht des europäischen Denkens zu verdichten und spürbare Lücken zu schließen. Einige wenige, aber prominente Artikel der alten Auflagen wurden völlig neu geschrieben. Daß bei diesen Absichten nicht alle Wünsche des Herausgebers in Erfüllung gegangen sind, sei nur am Rande erwähnt, waren unter anderem einige der angestammten Autoren nicht mehr zu erreichen.

Das Buch verwendet dennoch ein Porträt, um seine Absicht als historisches Lexikon zu verdeutlichen: Der Schutzumschlag zeigt den französischen Aufklärungsphilosophen Voltaire, den seine Zeitgenossen ziemlich realistisch als kecken, vornehmen Höfling porträtiert haben. In Horst Janssens Porträt, einer Mischtechnik von 1957, ist davon so gut wie nichts mehr zu erkennen. Dennoch ist es Voltaire, Janssens Voltaire. Über die Jahrhunderte, die uns samt unseren geschichtlichen Erfahrungen von der Französischen Revolution trennen, ist er gealtert, steckt in einer Art Kasperluniform, hat die Züge einer Karikatur angenommen, ist zur Unkenntlichkeit verkommen; die Amplituden von Nase und Zunge, die Janssen andeutet, haben beachtliche Dimensionen: Dialektik der Aufklärung, Identität des Nichtidentischen, Beharrlichkeit im Fortschritt, diese dubiose Signatur charakterisiert Janssens transformierender Zeitraffer, der für unsere Situation als ein wenig ratlose Erben der Aufklärung steht.

Auch dieses Buch rafft zweieinhalb Jahrtausende der philosophischen Verständigung über das Verhältnis von Mensch, Gott und Welt. Es ist aber ein historisches und kein systematisches Lexikon. Trotz allen Kulturwandels, der zwangsläufig in den Artikeln durchdringt, trotz aller Katastrophen, die Europa in diesem Zeitraum heimgesucht haben und die zu verkraften waren, ergibt sich am Ende vielleicht so etwas wie ein inhärenter Kern: $\mathrm{Da}$ das »Projekt Aufklärung « noch nicht an sein Ende gekommen ist, worin immer man dieses Ende erblicken mag. Vielleicht kommt es ja darauf an, die Sonderstellung des Menschen in der ihn umgebenden Welt nicht weiter auszubauen zu einer alles beherrschen Dominanz, die wir allenthalben als 
fortschreitenden und sich beschleunigenden Prozeß der Zivilisation bestaunen, sondern sie ebenso bestaunenswert - „vernünftig« zurückzubauen zu einer Weltgemäßheit des Menschen: »Wie weit es immer dem Menschen gelingen mag, sich die Natur durch Bearbeitung anzueignen und seine Herrschaft über sie auszudehnen, sie wird niemals zu unserer Umwelt, sie bleibt immer sie selbst. Von dieser Welt, die nicht eine Welt unter anderen und keine bloße Ideer (Kant) oder ein `Horizont`(Husserl) oder `Entwurf`(Heidegger) ist, sondern die eine und ganze wirkliche Welt, ließe sich sagen, daß über sie hinaus nichts Größeres denkbar ist. Sie braucht aber auch gar nicht als existierend bewiesen zu werden, den sie weist sich alltäglich und fortwährend selber aus, obwohl wir von unserer Weltgemäßheit zumeist so wenig wissen, wie die Zugvögel, die sich auf ihrem Flug am Stand der Sonne orientieren. Wir können keinen Augenblick existieren ohne die Welt, aber diese kann auch ohne uns sein" (Karl Löwith: Curriculum vitae, 1959). Ein Aufruf zu mehr Bescheidenheit, darin könnte unter anderem eine Reverenz des europäischen Denkens, das man auch das okzidentale, das abendländische genannt hat, an das östliche Denken bestehen, das hier nicht zur Darstellung kommen kann.

Schließlich: Dank allen, die zum Zustandekommen dieses Buchs beigetragen haben. - Solange sie Mühe machen, dauern die Werke.

Stuttgart, im Juli 2003

Bernd Lutz 